\title{
Robust Models for Location and Inventory Decisions in Emergency-grain Depots
}

\author{
Yu Fan, Can-Rong Zhang \\ Department of Industrial Engineering, Tsinghua University, Beijing, China \\ E-mail: fy15@mails.tsinghua.edu.cn, crzhang@sz.tsinghua.edu.cn
}

\begin{abstract}
The paper presents two robust models to solve the location-inventory problem occurring in the emergency grain depots (EGD) when demands are uncertain. The proposed model simultaneously determines the location of EGDs, the grain inventory at each employed EGD and the allocation of grain among emergency sites, with the objective of minimizing the system-wide cost which includes EGDs construction cost, grain inventory cost and grain transportation cost. Firstly, a mixed integer programming model is built for the deterministic problem. Secondly, two robust optimization models are established for the case when the demand volume of grain in each emergency site is uncertain. The two robust models, respectively, correspond to the Soyster [1] robust optimization model which is dedicated to the worst case, and the budget optimization model which was proposed by Brtsimas \& Sim [2] and has the advantage to control the robust level. Extensive numerical experiments are conducted to show the benefits of the two proposed robust optimization models. Interesting insights related to the problem are presented as well.
\end{abstract}

Keywords-location-inventory; uncertainty; robust optimization; emergency grain depots

\section{INTRODUCTION}

In recent years, unconventional emergency occurs frequently. It is of great significance for Chinese local government to optimize EGD construction and management. There are many uncertain factors in the EGD Locationinventory problem.

To capture the uncertainty in the emergency problem, Huseyin et al. [3] proposed a stochastic optimization method for the reserves and distribution in disaster cases. Carmen, Mark et al. [4] presented two stage stochastic mixed integers programming considering the uncertainty of hurricanes. Javier, Aruna [5] developed two stage stochastic optimization models to guide decisions before disaster. Araz et al. [6] proposed a fuzzy location model. The above researches on stochastic and fuzzy programming are limited in that:

1) The probability distribution of uncertain parameters in stochastic programming is difficult to be known;

2) Fuzzy programming has strong subjectivity to determine fuzzy membership function of uncertain parameters by a limited number of data samples and decision maker's experience.

To address the challenges, the paper introduces two robust optimization models to deal with the uncertainty. The main contributions of the paper are summarized as follows:

1) The paper introduces robust optimization models to the location-inventory EGD problem, in which the associated uncertainties are hard to quantify through probability distribution functions;

2) We provide two different robust approaches and compare their merits and drawbacks;

3) We conduct extensive numerical experiments and provide some interesting managerial insights to optimize the problem. The remainder of this paper is organized as follows: problem formulation is constructed in section 2; robust optimization approach formulation is given in section 3; numerical experiments are conducted in Section 4; and the conclusions are drawn in Section 5.

\section{PROBLEM FORMULATION}

\section{A. Problem Description}

We study the location and inventory problem of emergency grain depots (EGD). Two sites are considered: candidate EGDs and emergency sites. The goal is to determine the set of employed EGDs among the candidate sites, the grain inventory level of each EGD and the fraction of emergency demands satisfied by each employed EGD for each emergency site with minimum system cost. In our research, the cost considered include construction cost of located EGDs, grains inventory cost and transportation cost.

To model this problem, the following notation is used throughout this paper.

Sets and parameters:

I set of emergency sites, indexed by i

$J \quad$ set of candidate EGD, indexed by j

$f_{j} \quad$ construction cost at EGD j

$d_{i j} \quad$ distance between demand site i and EGD j

$C_{1} \quad$ unit transportation cost(unit volume, distance)

$C_{2} \quad$ unit inventory cost

$\mu_{i} \quad$ demand at demand site $\mathrm{i}$

Q maximum quantities of emergency sites served by each EGD

$G$ maximum quantities of grain inventory at each located EGD

$M \quad$ a sufficiently large number

Decision variables:

$X_{i j} \quad$ the fraction of demand at demand site i served by

EGD j

$Y_{j} \quad$ equals 1 , if candidate EGD $\mathrm{j}$ is located and 0 , otherwise

$S_{j} \quad$ quantity of grain inventory at EGD j 


\section{B. Nominal Problem Formulation}

In this section, the nominal problem of locationinventory EGD is formulated. All parameters in the problem are assumed to be deterministic and known in advance. The nominal model (NM) is formulated as follows:

$$
\begin{gathered}
\min \sum_{j \in J} f_{j} Y_{j}+\sum_{j \in J} \sum_{i \in I} c_{1} d_{i j} \mu_{i} X_{i j}+\sum_{j \in J} c_{2} S_{j} \\
\text { s.t. } \sum_{j \in J} X_{i j}=1, \forall i \in I \\
X_{i j} \leq Y_{j}, \forall i \in I, \forall j \in J \\
S_{j} \leq M Y_{j}, \forall j \in J \\
\sum_{i \in I} X_{i j} \leq Q, \forall j \in J \\
\sum_{i \in I} \mu_{i} X_{i j} \leq S_{j}, \forall j \in J \\
0 \leq S_{j} \leq G, \forall j \in J \\
0 \leq X_{i j} \leq 1, \forall i \in I, \forall j \in J \\
Y_{j} \in\{0,1\}, \forall j \in J
\end{gathered}
$$

The objective function (1) is to minimize the sum of construction cost for EGDs, transportation cost between EGDs and emergency sites, and grains inventory cost. Constraints (2) ensure that demands at each emergency site are served. Constraints (3) and (4) state that emergency sites and grains can only be assigned to employed EGDs. Constraints (5) stipulate that the number of emergency sites served by each EGD is no more than maximum capacity. Constraints (6) guarantee that there are sufficient grains to meet the demands assigned to each EGD. Constraints (7) and (8) represent the range of grains at each EGD and the fraction of demand at emergency site i served by EGD $j$, respectively. Constraints (9) are standard binary constraints.

\section{The Robust Optimization ApPROACH}

\section{A. Soyster Robust Optimization Model}

We assume the demands at emergency sites belongs to a box uncertainty set which is define as: $U^{B}=\left\{\mu_{i} \in R^{n}:\left|\mu_{i}-\overline{\mu_{i}}\right| \leq \widehat{\mu_{i}}, \forall \mathrm{i} \in I\right\}$, wheren $=|I|, \overline{\mu_{i}}$ is the nominal value of $\mu_{i}, \widehat{\mu}_{i}$ represents the uncertainty scale for this given entry. Under the box uncertainty set, the constraints (6) can be derived as follows: $\max _{\mu_{i} \in U^{B}}\left\{\sum_{i \in I} \mu_{i} X_{i j}\right\}=\sum_{i \in I}\left(\overline{\mu_{i}}+\widehat{\mu}_{i}\right) X_{i j} \leq S_{j}, \forall j \in J$; the last term of objective function (1) can be derived as follows: $\max _{\mu_{i} \in U^{B}}\left\{\sum_{j \in J} \sum_{i \in I} c_{1} d_{i j} \mu_{i} X_{i j}\right\}=\sum_{j \in J} \sum_{i \in I} c_{1} d_{i j}\left(\overline{\mu_{i}}+\widehat{\mu_{i}}\right)$.

As a result, the Soyster robust optimization model (ROM2) is formulated as follow:

$$
\min \sum_{j \in J} f_{j} Y_{j}+\sum_{j \in J} c_{2} S_{j}+\sum_{j \in J} \sum_{i \in I} c_{1} d_{i j}\left(\overline{\mu_{i}}+\widehat{\mu_{i}}\right) X_{i j}
$$

s.t. (2), (3), (4), (5), (7), (8), (9)

$$
\sum_{i \in I}\left(\overline{\mu_{i}}+\widehat{\mu_{i}}\right) X_{i j} \leq S_{j}, \forall j \in J
$$

\section{B. Budget Robust Optimization Model}

We assume the demands at emergency sites belongs to a polyhedron uncertainty set which is define as: $U_{p o l}=\left\{\mu_{i} \in \mathrm{R}^{n}\left|\sum_{i}\right| \mu_{i}-\overline{\mu_{i}}\left|/ \widehat{\mu}_{i} \leq \Gamma,\right| \mu_{i}-\overline{\mu_{i}} \mid / \widehat{\mu}_{i} \leq 1, \forall i \in I\right\}$ . We define the budget of uncertainty $\Gamma$ as the RO approach proposed by Bertsimas and Sim [2] to control the degree of robustness and conservativeness of the solution takes values in the interval $[0,|I|]$. The parameter $\Gamma$ is not necessarily integer which indicates that up to $\lfloor\Gamma\rfloor$ emergency sites are allowed to have uncertain demands, and one emergency site changes demands by $(\Gamma-\lfloor\Gamma\rfloor) \hat{\mu}$.

For the objective function (1), the nonlinear robust formulation is as follows:

$$
\begin{gathered}
\min \left\{\sum_{j \in J} f_{j} Y_{j}+\sum_{j \in J} c_{2} S_{j}+\sum_{j \in j \in l \in I} c_{1} d_{i j} \bar{\mu}_{i} X_{i j}+\max \left\{\sum_{j \in j} \sum_{i \in l} c_{1} d_{i j} \hat{\mu_{i}} X_{i j} \xi_{i}\right\}\right\} \\
\sum_{i \in I} \xi_{i} \leq \Gamma \\
0 \leq \xi_{i} \leq 1, \forall i
\end{gathered}
$$

The equivalent linear formulation for above model is as follows:

$$
\begin{gathered}
\min \left\{\sum_{j \in J} f_{j} \mathrm{Y}_{j}+\sum_{j \in J} c_{2} S_{j}+\sum_{j \in J} \sum_{i \in I} c_{1} d_{i j} \bar{\mu}_{i} X_{i j}+\mathrm{z} \Gamma+\sum_{i \in I} \delta_{i}\right\} \\
\mathrm{z}+\delta_{i} \geq \widehat{\mu_{i}} \sum_{j \in J} c_{1} d_{i j} X_{i j}, \forall i \in I \\
z, \delta_{i} \geq 0, \forall i \in I
\end{gathered}
$$

Where $z, \delta_{i}$ are dual variables for constraints (13), constraints (14), respectively. 
Similarly, the nonlinear robust formulation of constraints (6) is as follows:

$$
\begin{gathered}
\left.\sum_{i \in I} \overline{\mu_{i}} X_{i j}+\max \left\{\sum_{i \in I} \widehat{\mu_{i}} X_{i j} \xi_{i}\right\}\right\} \leq S_{j}, \forall j \in J \\
\sum_{i \in I} \xi_{i} \leq \mathbf{I} \\
\mathbf{O} \leq \xi_{i} \leq \mathbf{1} \forall \boldsymbol{i}
\end{gathered}
$$

The equivalent linear formulation for above model is as follows:

$$
\begin{gathered}
\max \sum_{i \in I} \overline{\mu_{i}} X_{i j}+\mathrm{l}_{j} \Gamma+\sum_{i \in I} \varepsilon_{i j} \leq S_{j}, \forall j \in J \\
l_{j}+\varepsilon_{i j} \geq \widehat{\mu}_{i} X_{i j}, \forall j \in J, \forall i \in I \\
l_{j}, \varepsilon_{i j} \geq 0, \forall i \in I, \forall j \in J
\end{gathered}
$$

Where $l_{j}, \varepsilon_{i j}$ are dual variables for constraints (19), constraints (20), respectively. As a result, the budget robust optimization model is formulated as follows:

$$
\begin{gathered}
\min \left\{\sum_{j \in J} f_{j} \mathrm{Y}_{j}+\sum_{j \in J} c_{2} S_{j}+\sum_{j \in J} \sum_{i \in I} c_{1} d_{i j} \overline{\mu_{i}} X_{i j}+\mathrm{z} \Gamma+\sum_{i \in I} \delta_{i}\right\} \\
\text { s.t. (2), (3), (4), (5), (7), (8), (9) } \\
\mathrm{z}+\delta_{i} \geq \widehat{\mu_{i}} \sum_{j \in J} c_{1} d_{i j} X_{i j}, \forall i \\
\sum_{i \in I} \overline{\mu_{i}} X_{i j}+l_{j} \Gamma+\sum_{i \in I} \varepsilon_{i j} \leq S_{j}, \forall j \in J \\
l_{j}+\varepsilon_{i j} \geq \widehat{\mu_{i}} X_{i j}, \forall j \in J, \forall i \in I \\
z, l_{j}, \delta_{i}, \varepsilon_{i j} \geq 0, \forall i \in I, \forall j \in J
\end{gathered}
$$

\section{NUMERICAL EXPERIMENTS}

In this section, we firstly randomly generate input data for the problem. Secondly, we generate solutions for above three models. Finally, we conduct numerical experiments to evaluate the solution and verify the benefits of our robust optimization models.

\section{A. Data Generation}

The values of parameter and information of random data generation are shown in Table1and Table II, respectively.
The $\mathrm{x}$ coordinate, $\mathrm{y}$ coordinate of emergency sites and candidate EGDs are generated randomly with uniform distribution $(0,100)$. The demands of each emergency site are generated randomly with uniform distribution $(100,150)$.

\begin{tabular}{cc} 
TABLE I. & VALUES OF PARAMETERS \\
\hline Parameter & Values \\
\hline$f_{j}$ & 500 \\
$C_{1}$ & 2 \\
$C_{2}$ & 200 \\
$Q$ & 6 \\
$S$ & 600 \\
$|I|$ & 40 \\
$|J|$ & 15 \\
$\Gamma$ & {$[0,40]$} \\
$\widehat{\mu_{i}}$ & 80 \\
\hline
\end{tabular}

\section{B. Solution Generation}

We program in Java and call CPLEX12.6 on eclipsemars to solve the models and conduct computational experiments. The operation environment is Windows7, RAM (4G), CPU (Intel(R), Pentium(R), CPU B940).Table III shows the objective values and run time of deterministic model (DM), Soyster robust optimization model (SM) and budget robust optimization model (BM).

TABLE II. RESULTS OF THREE MODELS

\begin{tabular}{ccc}
\hline Model & Optimal Value & CPU time \\
\hline $\mathrm{DM}$ & 1122229.245 & $0.263 \mathrm{~s}$ \\
$\mathrm{SM}$ & 1864251.622 & $0.146 \mathrm{~s}$ \\
$\mathrm{BM}(\Gamma=4)$ & 1452585.397 & $0.258 \mathrm{~s}$
\end{tabular}

The results show that when given the nominal demands, the deterministic model provides solution of minimum system cost; the Soyster robust optimization solves the location-inventory EGD problem at highest cost because it considers the worst case of uncertain demands; the budget robust optimization model has the media system cost, due to its trade-off between the robustness and total cost. The results are in line with our former theoretical derivations.

\section{Solution Evaluation}

To evaluate the solution for each model, we conduct extensive numerical experiments. We generate 100 samples in which demands vary in range $\left[\overline{\mu_{i}}-\widehat{\mu_{i}}, \overline{\mu_{i}}+\widehat{\mu_{i}}\right]$ to simulate the real emergency situation. The solution performances are evaluated by the mean of objectives values, standard variance of objective values (SD) and constraints violation percentages. The solution evaluation is shown in Table III. 
TABLE III. SOLUTION EVALUATION OF THREE MODELS

\begin{tabular}{cccc}
\hline Model & Mean of Objectives Values & SD & $\begin{array}{c}\text { Constraints } \\
\text { Violation } \\
\text { Percentages }\end{array}$ \\
\hline DM & 1122246.349 & 7497.418 & $46.56 \%$ \\
SM & 1864251.622 & 8675.450 & $0 \%$ \\
$\mathrm{BM}(\Gamma=4)$ & 1452585.397 & 15437.122 & $3.4 \%$ \\
\hline
\end{tabular}

The results show that the deterministic model solution is unacceptable in real situations since it causes nearly a half of constraint violation. Robust optimization models are applicable to cope with uncertain demands in emergencies. Specifically, the Soyster robust optimization model ensures $100 \%$ service level and causes highest total cost; and Budget robust optimization model ( $\Gamma=4$ ) achieves a quite high service level(not 100\%) and incurs relatively lower cost.

\section{Comparisons of the Models}

In this section, we generate solutions for Budget robust optimization model by varying the values of parameter $\Gamma$ and observe solution performance. The results are show in Table IV.

TABLE IV. SOLUTION PERFORMANCE OF BUDGET MODEL

\begin{tabular}{cccc}
\hline$\Gamma$ & $\begin{array}{c}\text { Mean of } \\
\text { Objectives Values }\end{array}$ & SD & $\begin{array}{c}\text { Constraints Violation } \\
\text { Percentage }\end{array}$ \\
\hline 0 & 1122246.349 & 7497.417 & $46.56 \%$ \\
4 & 1428288.259 & 15437.122 & $3.48 \%$ \\
8 & 1547963.604 & 18345.525 & $0.29 \%$ \\
12 & 1642969.380 & 18362.013 & $0.013 \%$ \\
14 & 1675735.295 & 18244.759 & $0 \%$ \\
20 & 1764630.011 & 10198.457 & $0 \%$ \\
30 & 1781945.910 & 8654.123 & $0 \%$ \\
40 & 1781404.636 & 8675.450 & $0 \%$ \\
\hline
\end{tabular}

The results show that when parameter $\Gamma$ increases slightly, the constraints violation percentages reduce significantly. Especially, when it takes value larger than 13, the constraints violation percentage decreases to zero.

We compare the mean of objective functions and constraints violation percentages between Soyster robust optimization model and budget robust optimization model. The comparisons are shown in Table V.
TABLE V. COMPARISONS RESULTS OF TWO MODELS

\begin{tabular}{ccc}
\hline Model & Mean of Objectives Values & $\begin{array}{c}\text { Constraints Violation } \\
\text { Percentage }\end{array}$ \\
\hline $\mathrm{SM}$ & 1781404 & 0 \\
$\mathrm{BM}(\Gamma=14)$ & 1675735 & 0 \\
$\mathrm{BM}(\Gamma=20)$ & 1764630 & 0 \\
$\mathrm{BM}(\Gamma=30)$ & 1781945 & 0 \\
$\mathrm{BM}(\Gamma=40)$ & 1781404 & 0 \\
\hline
\end{tabular}

The results show that when $\Gamma$ changes from 14 to 40 , the Budget robust optimization model ensures $100 \%$ service level as well as Soyster robust optimization model. Meanwhile the Budget robust optimization model has lower system cost than Soyster robust optimization model.

\section{CONCLUSIONS}

In the paper, we introduce two robust optimization models to location-inventory problem of EGDs. Extensive numerical experiments are conducted to verify the applicability of robust models. We have following conclusions: 1) The deterministic model is not applicable to real emergencies; 2)To ensure 100\% service level, Soyster robust optimization model is preferable and it incurs higher system cost; 3)Budget robust optimization model makes balance between service level and total cost by varying the budget of robustness. We suggest the following future research directions: develop new robust optimization models under different uncertainty sets such as ellipsoid uncertainty sets.

\section{ACKNOWLEDGEMENT}

This work is supported by the National Nature Science Foundation of China under Grant no. 71472108.

\section{REFERENCES}

[1] Soyster AL., "Convex Programming with set inclusive constraints and applications to inexact linear Programming”, Operations Research, pp. 1154-1157, 1973.

[2] Bertsimas, D., \& Sim, M. (2004). The price of robustness. Operations research, vol.52, no.1, pp.35-53.

[3] Huseyin Onur Mete, Zelda B, Zabinsky, "Stochastic optimization of medical supply location and distribution in disaster management”, Int J Production Economics, vol.126, pp. 76- 84, 2010.

[4] Carmen G Rawls, Mark A Turnquist, "Pre-positioning planning for emergency response with service quality constraints", OR Spectrum, vol.33, pp.481- 498, 2011.

[5] Javier Salmeron, Aruna Apte, "Stochastic optimization for natural disaster asset prepositioning", Production and operations management, vol.19, no.5, pp. 561- 574, 2010.

[6] C. Araz, H. Selim, I. Ozkarahan, "A fuzzy multi-objective coveringbased vehicle location model for emergency services”, Comput. Oper. Res. Vol.34 ,no.3, pp.705-726, 2007. 